\title{
LECTURE 14
}

\section{Scaling in stock market data: stable laws and beyond}

\author{
Rama $\operatorname{CONT}\left({ }^{1,2,3}\right)$, Marc POTTERS $\left({ }^{2}\right)$ \\ and Jean-Philippe BOUCHAUD $\left({ }^{1,2}\right)$ \\ (1) Service de Physique de l'Etat Condensé \\ Centre d'Etudes de Saclay 91191 Gif-sur-Yvette, FRANCE \\ $\left.{ }^{2}\right)$ Science \& Finance \\ 109-111 rue Victor Hugo \\ 92532 Levallois, FRANCE \\ $\left(^{3}\right)$ Laboratoire de Physique de la Matière Condensée \\ CNRS URA 190, Université de Nice \\ 06108 Nice Cedex 2, France
}

\section{Introduction}

The concepts of scale invariance and scaling behavior are now increasingly applied outside their traditional domains of application, the physical sciences. Their application to financial markets, initiated by Mandelbrot $[1,2]$ in the 1960s, has experienced a regain of interest in the recent years, partly due to the abundance of high-frequency data sets and availability of computers for analyzing their statistical properties.

This lecture is intended as an introduction and a brief review of current research in a field which is becoming increasingly popular in the theoretical physics community. We will try to show how the concepts of scale invariance and scaling behavior may be usefully applied in the framework of a statistical approach to the study of financial data, pointing out at the same time the limits of such an approach. 


\section{Statistical description of market data}

Quantitative research mainly focuses on "liquid" financial markets i.e. organized markets where transactions are frequent and the number of actors is large. Typical examples are foreign exchange markets, organized futures markets and stock index markets and the market for large stocks. Prices are recorded several times a minute in such markets, creating mines of data to exploit. Such markets are complex systems with many degrees of freedom [3], where many internal and external factors interact at each instant in order to fix the transaction price of financial assets.Various factors such as public policy, interest rates and economic conditions doubtlessly influence market behavior. However, the precise nature of their influence is not well known and given the complex nature of the pricing mechanism, simple deterministic models are unable to reproduce the properties observed in financial time series. Furthermore, although the details of the price fixing mechanism- market microstructure - may be different from one market to the other, what is striking is the universality of some simple statistical properties of price fluctuations, prompting a unified approach to the study of different types of markets.

As in the case of other types of complex systems with universal characteristics, a stochastic approach has proved to be fruitful in this case. The main object of study in this framework is the probability density function (PDF) of the increments at a given time scale $T$ i.e. the probability distribution $P_{T}$ of $x(t+T)-x(t)$ where $x(t)$ is the price of the asset at time $t$. This approach was inaugurated by Louis Bachelier who first introduced the idea that stock market prices behave as a random walk [4], and who considered Brownian motion as a candidate for modeling price fluctuations.

Bachelier's model, applied to the logarithm of the prices (to ensure positivity of the price!), became very popular in the 1950s [5] and it is one of the main ingredients of the famous Black-Scholes option pricing formula [6]. This model implies that the increments of asset returns (or asset prices) are independent identically distributed (iid) Gaussian variables. Indeed if one considers each price change as a sum of many small and independent random contributions from various market factors, the Central Limit Theorem suggests the Gaussian as a natural candidate.

Figure 1. gives a typical example of the empirical distribution of the increments of asset prices, in this case the U.S. Dollar/ Deutschemark exchange rate, sampled every 5 minutes. The contrast with the Gaussian is striking: this 'heavy tailed' or 'leptokurtic' character of the distribution of price changes, has been repeatedely observed in various market data and may be quantitavely measure by the kurtosis of the distribution $P_{T}$ defined by :

$$
\kappa=\frac{\overline{\left(\Delta_{T} x-\overline{\Delta_{T} x}\right)^{4}}}{\sigma(T)^{4}}-3
$$

where $\sigma(T)$ is the variance of $\Delta_{T} x=x(t+T)-x(t)$. The kurtosis is defined such 
that $\kappa=0$ for a Gaussian distribution, a positive value of $\kappa$ indicating a slow asymptotic decay of the PDF. The kurtosis of the increments of asset prices is far from its Gaussian value: typical values for $\mathrm{T}=5$ minutes are: $\kappa \simeq 74$ (US\$/DM exchange rate futures), $\kappa \simeq 60$ (US\$/Swiss Franc exchange rate futures), $\kappa \simeq 16$ (S\&P500 index futures) $[7,8]$.

These observations imply that using a Gaussian PDF systematically underestimates the probability of large price fluctuations, an issue of utmost importance is financial risk management.

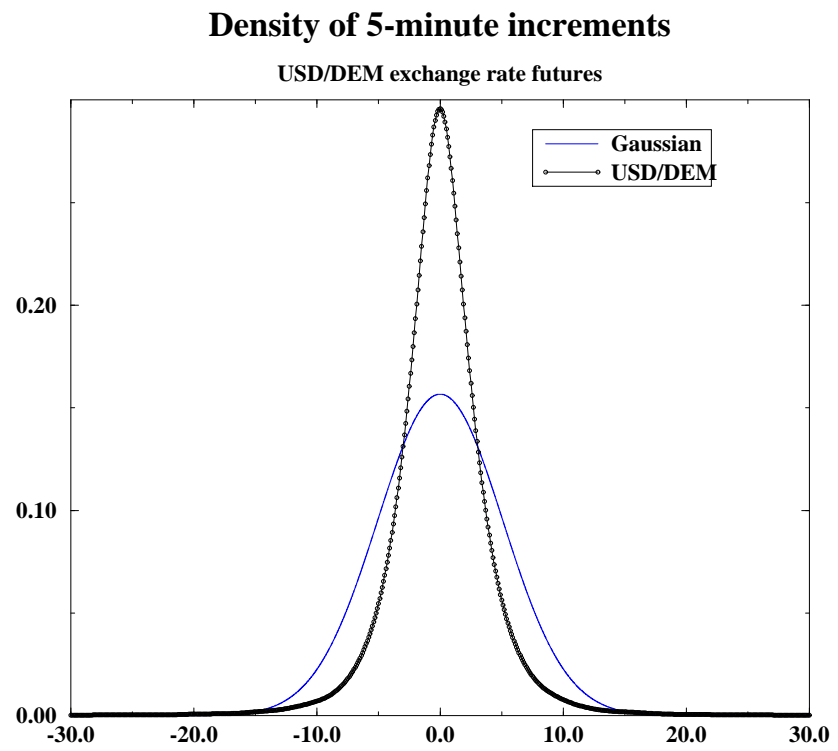

Fig. 1. - Probability density of 5 minute increments of USD/Deutschemark exchange rate futures. The lower curve is a gaussian with same mean and variance.

\section{Scale invariance and stable laws}

But why should stock price fluctuations have scale invariant properties in the first place? The answer lies in a generalized version of the Central Limit Theorem [9]: the distribution of the sum a large number of independent identically distributed random variables belongs to a family of distributions known as stable or stable Lévy distributions, characterized by their Fourier transform (characteristic function) $[9,10]$ :

$$
\phi_{\mu}(z)=\exp \left[-a_{\mu}|z|^{\mu}\left(1+i \beta \tan (\mu \pi / 2) \frac{z}{|z|}\right)\right]
$$

The parameter $\mu$, called the stability index, belongs to the interval $] 0,2], \mu=2$ corresponding to the Gaussian distribution. $\beta$ is a skewness parameter, $\beta=0$ for a symmetric distribution. Lévy distributions are characterized by the property of being stable under convolution: in other words, the sum of two iid Lévy-distributed 
variables is also Lévy distributed, with the same stability index $\mu$. More precisely, if $X_{i}, i=1 . . n$ are $\mathrm{n}$ iid $\mu$-stable random variables then the renormalized sum:

$$
S_{n}=\frac{\sum_{i=1}^{n} X_{i}}{N^{1 / \mu}}
$$

has the same PDF as the $X_{i}$. In particular, the sum scales as $N^{1 / \mu}$ and not as $\sqrt{N}$ which is the case in diffusive random walks. Lévy distributions, in addition to their stability under convolution, have other interesting properties: except for the Gaussian $(\mu=2)$ all $\mu$-stable PDFs have power-law tails with exponent $1+\mu$ :

$$
p_{\mu}(x) \simeq \frac{C}{x^{1+\mu}}
$$

leading to an infinite variance and heavy tails.

These observations led Mandelbrot to propose stable Lévy distributions as candidates for the PDF of price changes of financial assets [1,2]. Mandelbrot observed that stable distributions offer a heavy tailed alternative to Bachelier's model while still enabling a justification of the model in terms of the Central Limit Theorem (see above). Furthermore, their stability under convolution gives rise to the scale invariance of the process: if appropriately rescaled, the increment at scale $N \tau$ will have the same distribution as the increment at scale $\tau$ :

$$
P_{N \tau}(x)=\frac{1}{\lambda} P_{\tau}\left(\frac{x}{\lambda}\right), \quad \lambda=N^{1 / \mu}
$$

The above relation means that the price process $x(t)$ is self-similar with a selfsimilarity exponent which is the inverse of the stability index $\mu$. Self-similarity in market prices was first remarked by Mandelbrot in the 1960s in his seminal work cotton prices [1]. Recent studies have confirmed the presence of self-similarity and scale invariant properties in various markets: the Milan stock exchange [11], the S\&P500 index [12], the CAC40 (Paris stock market) index [13] and foreign exchange markets $[14,15]$ and individual french stocks [16]. The value of $\mu$ may be estimated for example by examining the scaling behavior of the probability of return to the origin $P_{T}(0)$, which scales as $\frac{1}{N^{1 / \mu}}$, which is indeed the method used in $[12,13]$. The value of $\mu$ found depends on the market considered but all values fall between 1.5 and 1.7, $\mu$ being lower for more volatile markets.

\section{Beyond scale invariance: truncated Lévy flights}

Although the representation of price increments as Lévy-stable iid variables accounts for their leptokurtic character as well as their self-similar properties, it has several drawbacks: careful comparison of the distributions at various time scales show that in fact it is not scale invariant $[8,15,7]$. The self-similarity properties described above do not hold at all time scales but only for short ones, typically less than a week. For example, if the price followed a Lévy walk, different methods of estimating the selfsimilarity parameter would give the same value, namely $1 / \mu$. Yet in practice, data from different time resolutions give different values of the self-similarity exponent, between 0.5 (the value for a Brownian diffusion) and $0.6(=1 / \mu$ with $\mu=1.7)$. 
Variance of price increments

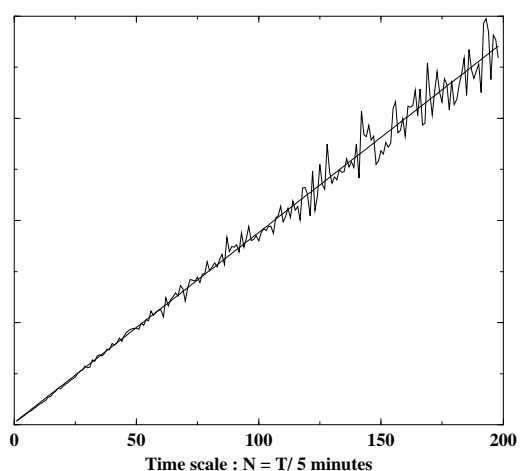

Scaling behavior of kurtosis

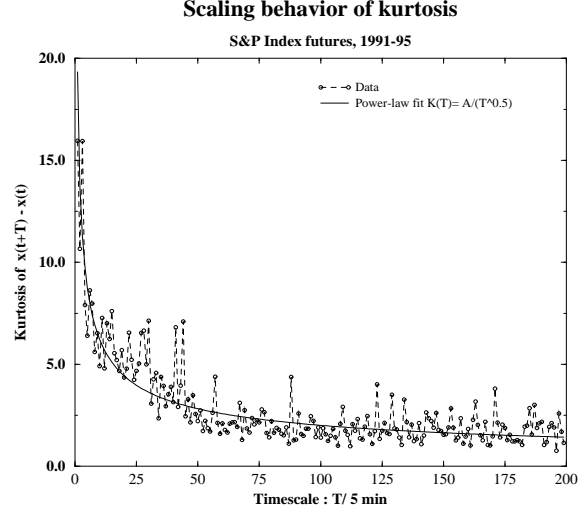

Fig. 2. - Left: Scaling behavior of the variance of price increments for $\mathrm{S} \& \mathrm{P} 500$ index futures: the variance increases linearly with the time scale, a property which is consistent with the absence of significant linear correlation. Right: Scaling behavior of the kurtosis of price increments for S\&P 500 index futures: the kurtosis decreases more slowly than in the case of iid increments where it decreases as $1 / N$. The solid line represents a fit with $\kappa(T)=\kappa(1) / \sqrt{T}$.

Furthermore, the best fit by a stable Lévy distribution [12] still overestimates the probability for extreme fluctuations: in other words, Lévy distributions have tails that are 'too fat' compared to fluctuations of real prices. Tails of empirical distributions may be studied more closely by rank-ordering techniques [8,17] (also called Zipf plots). They turn out to decay more slowly than a Gaussian but more quickly than any Lévy-stable distribution: their decay is better described by an exponential tail. Last but not least, real price changes turn out to have finite variance $[8,15]$.

These apparently contradictory aspects- self-similarity at short time scales, breakdown of scaling for longer time scales, truncated power law tails, finite variance- may be blended together into a consistent picture by a truncated Lévy flight description $[18,8,19]$. Consider a random walk with increments distributed with an exponentially truncated Lévy density $p(x)$ [20]. $p(x)$ may be characterized by its Fourier transform (characteristic function):

$$
\phi(z)=\exp \left(-a_{\mu}\left[\left(\left(\alpha^{2}+z^{2}\right)^{\mu / 2} \frac{\cos (\mu \arctan (|z| / \alpha))}{\cos (\pi \mu / 2)}-\alpha^{\mu}\right)\right]\right)
$$

The above expression differs from the characteristic function of a stable law by the presence of a cut-off parameter $\alpha . \phi(z)$ is defined such that it has a regular Taylor expansion at the origin, assuring the finiteness of all moments. In terms of the variable $x, p(x)$ decays exponentially for large values of $x$, which describes adequately the decay of the tails of the PDF of empirical price changes. The asymptotic behavior of $\phi$ is given by

$$
\phi(z) \underset{z \rightarrow \infty}{\sim} \phi_{\mu}(z)
$$

i.e. for small values of $x, p(x)$ behaves like a Lévy-stable law of index $\mu$. We can now proceed to describe the behavior of a random walk with iid increments having 
a truncated Lévy PDF $p(x)$. For short time scales, $p(x)$ behaves like a stable law of index $\mu$ : the short term dynamics of the process is therefore correctly described by a Lévy flight which gives rise to the scale invariance with a self-similarity exponent $H=1 / \mu[1,2,11,12]$. However, since the variance of the distribution is finite, the Gaussian version of the Central Limit Theorem applies and for large time scales $T \gg T^{*}=\kappa \tau$, where $\kappa$ is the kurtosis of the distribution $P_{\tau}(x)$. Thus at large time resolutions the distribution $P_{T}$ will approach a Gaussian, which is consistent with the fact that the kurtosis tends to zero when $T \rightarrow \infty$ (see figure). Between these two regimes -Lévy flight for short times and Gaussian diffusion for long times- is a crossover regime which is characterized by the appearance of fluctuations of the order $1 / \alpha$.

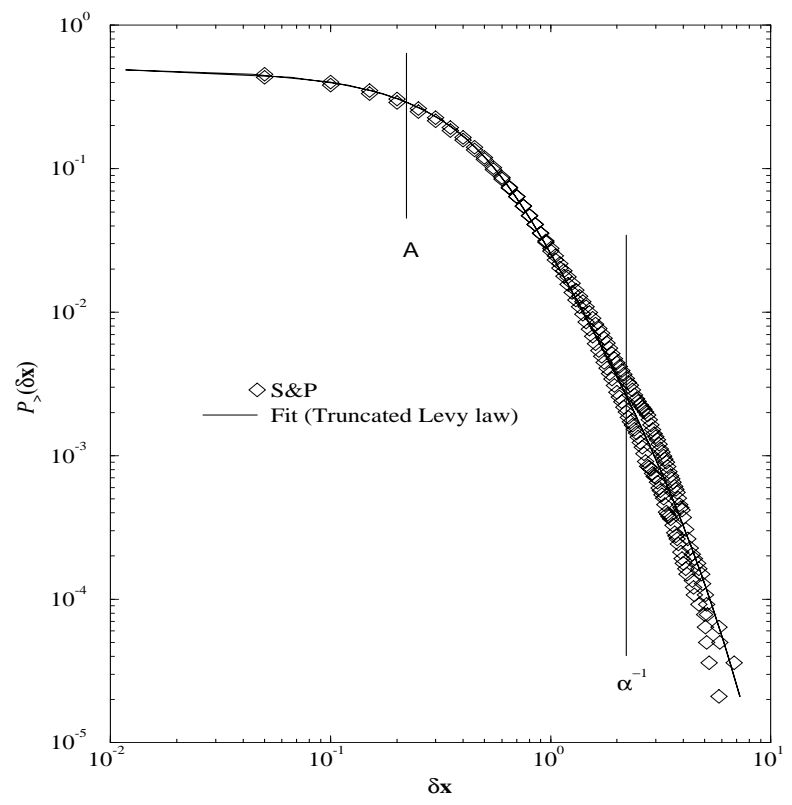

Fig. 3. - Rank ordering of the increments of S\&P 500 index futures. The cumulative distribution function of price increments is well represented by an exponentially truncated Lévy distribution with finite variance.

\section{Correlation and dependence}

It is a well-known fact that price movements in liquid markets do not exhibit any significant autocorrelation: the autocorrelation function of the price changes 


$$
C(T)=\frac{\overline{\delta x_{t} \delta x_{t+T}}-\overline{\delta x_{t}} \overline{\delta x_{t+T}}}{\operatorname{var}\left(\delta x_{t}\right)}
$$

rapidly decays to zero in a few minutes: for $T \geq 15$ minutes it can be safely assumed to be zero for all practical purposes [19]. The absence of significant linear correlations in price increments and asset returns has been widely documented [21, 22] and often cited as support for the "Efficient Market Hypothesis" [23]. The absence of correlation is intuitively easy to understand: if price changes exhibit significant correlation, this correlation may be used to conceive a simple strategy with positive expected earnings; such strategies, termed arbitrage, will therefore tend to reduce correlations except for very short time scales, which represent the time the market takes to react to new information. This correlation time is typically several minutes for organized futures markets and even shorter for foreign exchange markets.

The fast decay of the correlation function implies the additivity of variances: for uncorrelated variables, the variance of the sum is the sum of the variances. The absence of linear correlation is thus consistent with the linear increase of the variance with respect to time scale.

However, the absence of serial correlation does not imply the independence of the increments [7]: for example the square or the absolute value of price changes exhibits slowly decaying serial correlations. Figure 5 displays the autocorrelation function $g(T)$ of the square of the increments, defined as:

$$
g(T)=\frac{\overline{\delta x_{t}^{2} \delta x_{t+T}^{2}}-\overline{\delta x_{t}^{2}} \overline{\delta x_{t+T}^{2}}}{\operatorname{var}\left(\delta x^{2}\right)}=\frac{\overline{\delta x_{t}^{2} \delta x_{t+T}^{2}}-\overline{\delta x_{t}^{2}} \overline{\delta x_{t+T}^{2}}}{\mu_{4}(\tau)-\sigma(\tau)^{4}}
$$

for S\&P 500 Futures.The slow decay of $g(T)$ (see figure) is well represented by a power law [7]:

$$
g(k) \simeq \frac{g_{0}}{k^{\alpha}} \quad \alpha=0.37 \pm 0.037 \quad g_{0}=0.08
$$

This slow relaxation of the correlation function $g$ indicated persistence in the scale of fluctuations. Other measures of the scale of fluctuations, such as the absolute value of the increments, exhibit the same type of persistence, a phenomenon which can be related to the "clustering of volatility", well known in the financial literature: a large price movement tends to be followed by another large price movement but not necessarily in the same direction. 

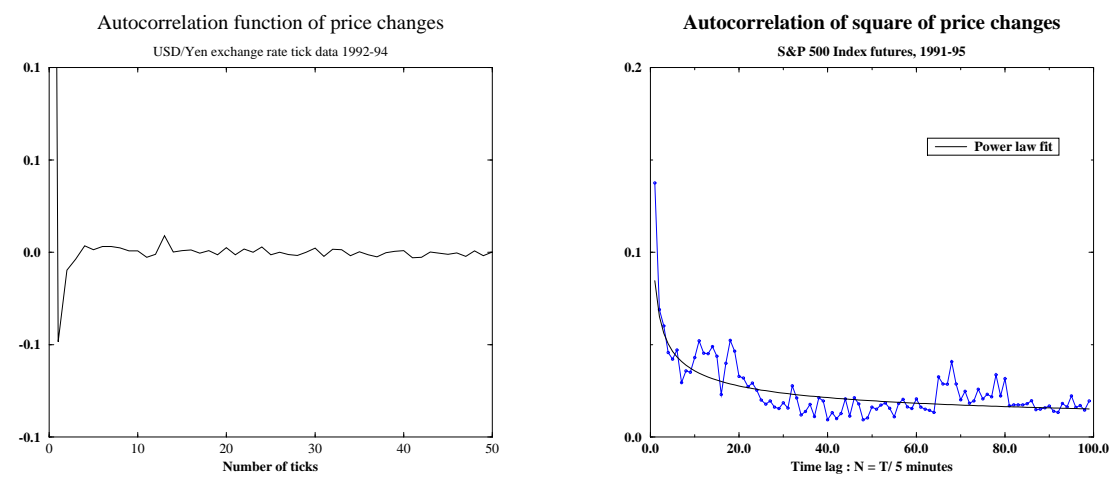

Fig. 4. - Left: Autocorrelation function of price increments for the US Dollar/ Yen exchange rate. Right: The autocorrelation function $g$ of square of price increments of $\mathrm{S} \& \mathrm{P}$ futures exhibits a slow decay, well represented by a power law with exponent $\alpha \simeq 0.37$.

The persistent character of the scale of fluctuations may be quantitatively related to the anomalous scaling behavior i.e. slow decrease of the kurtosis as the time resolution increases. As a result, the convergence to the Gaussian is slowed down even more than in the case of a truncated Lévy flight with iid increments.

The presence of such subtle correlations points out the limits of a representation of market prices by a random walk model and the need to take into account finer effects due to nonlinear correlations and non-stationarity [24,7]. These effects are especially important in options markets, where the behavior of volatility -the scale of price fluctuations- is crucial in determining the value of the option. It has been shown [24] that the correlations present in the volatility -as represented by the function $g$ defined above- are accurately reflected in market prices for options.

\section{Turbulence and finance}

Recently some attempts have been made to draw analogies between scaling properties of foreign exchange rates and turbulent flows [25], based on some similarities between their probability density functions. Indeed, the statistical approach to the problem is the same, price increments playing the same role as velocity increments in the case of turbulence and a formal analogy may be very tempting. However, in the light of the above remarks, it is clear that such an analogy cannot be pushed too far: there is an essential difference between the two phenomena, namely, the presence of strong correlations between velocity increments, leading to Kolmogorov's famous $k^{-5 / 3}$ law [26], which are absent in the case of price increments [19]. As remarked above, the autocorrelation function of price increments decreases rapidly to zero, resulting in a flat ("white-noise") spectrum (see figure below, taken from [19]). Translating into a power spectrum we obtain a dependence well approximated by $\omega^{-2}$, which merely reflects the absence of correlation, as opposed to the $\omega^{-5 / 3}$ law for turbulent flows. 


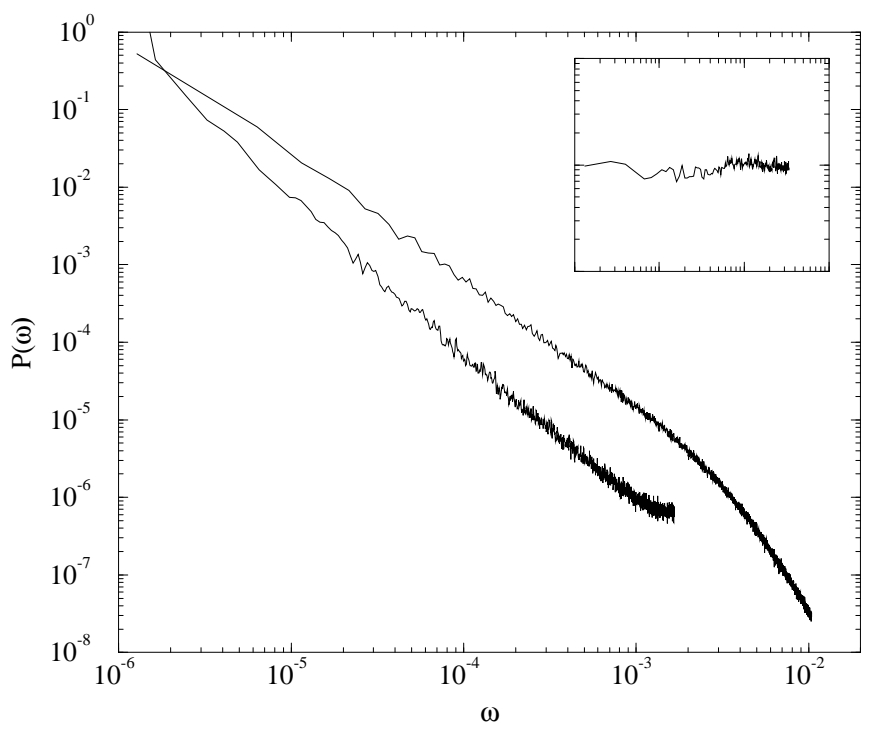

Fig. 5. - Fourier transform of the price autocorrelation function $\langle x(t) x(t+\tau)\rangle$ as a function of temporal frequency for the DEM-USD exchange rate (Oct 1991-Nov 1994) (bottom curve) as compared to the $k^{-5 / 3}$ power-law spectrum observed for the spatial velocity fluctuations in turbulent flows (the data were recorded by Y. Gagne in a wind tunel experiment at $R_{\lambda}=3050$ ) (top curve). Inset: Fourier transform of the price change autocorrelation function $\langle\Delta x(t) \Delta x(t+\tau)\rangle$, which is completely flat (and would behave as the $1 / 3-t h$ power of frequency in turbulent flows). Units are arbitrary.

\section{Conclusion}

The above example illustrates that although methods of statistical physics and in particular the concepts of scaling and scale invariance may be successfully applied in the study of market price fluctuations, sometimes revealing unsuspected properties of financial time series, analogies with problems in physics should be handled with care in order to avoid erroneous conclusions. Keeping this point in mind, the scientific study of financial markets proves to be a fascinating subject in itself, in particular for the physicist whose theoretical tools may prove to be useful in uncovering new properties and mechanisms in financial data.

- Acknowledgements:

We thank J.P. Aguilar for his helpful remarks. 


\section{References}

[1] Mandelbrot, B. (1963) "The variation of certain speculative prices" Journal of Business, XXXVI, 392-417.

[2] Mandelbrot, B. \& Taylor, H.M. (1967) "On the distribution of stock price differences" in Operations Research,15, 1057-1062.

[3] Pines, D. et al. (eds.) The Economy as an evolving complex system Santa Fe Institute, Addison-Wesley.

[4] Bachelier, L. (1900) "Théorie de la spéculation " Annales Scientifiques de l'Ecole Normale Supérieure, III : 17, 21-86. Bachelier's seminal work is also the first mathematical study of Brownian motion, preceding Einstein's famous work on the same subject.

[5] Cootner, P. (ed.) The random character of stock market prices Cambridge, MA: The MIT Press.

[6] Black, F. \& Scholes, M. (1973) "The pricing of options and corporate liabilities" Journal of Political Economy, 81, 635-654.

[7] Cont, R. (1997) "Scaling and correlation in financial time series" Science \& Finance Working Paper 97-01 (cond-mat/9705075).

[8] Bouchaud, J.P. \& Potters, M. (1997) Théorie des risques financiers Paris: Aléa Saclay (forthcoming).

[9] Gnedenko, B.V. \& Kolmogorov, A.N. (1954) Limit distributions for sums of independent random variables, Addison-Wesley, Reading, MA.

[10] Bouchaud, J.P. \& A. Georges (1990) Physics Reports 195, 125.

[11] Mantegna, R.N. (1991) "Lévy walks and enhanced diffusion in the Milan stock exchange" Physica A, 179, 232.

[12] Mantegna, R.N. \& Stanley, H.E. (1995) "Scaling behavior of an economic index" Nature, 376, 46-49.

[13] Zajdenweber, D. (1994) "Propriétés autosimilaires du CAC40" Revue d'Economie Politique, 104, 408-434.

[14] Pictet O.V. et al. (1995) "Statistical study of foreign exchange rates, empirical evidence of a price change scaling law and intraday analysis" Journal of Banking and Finance, 14, 1189-1208.

[15] Guillaume, D.M. et al. "From the bird's eye to the microscope: a survey of new stylized facts of the intra-day foreign exchange markets" Olsen \& Associates Research Group working paper.

[16] Belkacem, L. (1996) Processus stables et applications à la finance, Thèse de Doctorat, Université Paris IX.

[17] Cont, R. (1997) Dynamical models of financial markets (Doctoral thesis), Université de Paris XI.

[18] Mantegna, R.N. \& Stanley, H.E. (1994) Phys. Rev. Lett., 73, 2946.

[19] Arnéodo,A. et al. (1996) "Comment on Turbulent Cascades in Foreign Exchange Markets" (preprint cond-mat/9607120) Science \&s Finance Working Paper 96-01.

[20] Koponen, I. (1995) Phys. Rev. E 52, 1197.

[21] Fama, E.F. (1970) "Efficient capital markets: review of theory and empirical work" Journal of Finance,25, 383-417.

[22] Pagan, A. (1996) "The econometrics of financial markets" Journal of Empirical Finance,3, 15-102. 
[23] Fama, E.F. (1965) "The behavior of stock market prices" Journal of Business, 38, 34-105.

[24] Potters, M., Cont, R. \& Bouchaud, J.P. (1996) "Financial markets as adaptive ecosystems" (preprint cond-mat/9609172) Science \& Finance Working Paper 96-02.

[25] Ghashghaie, S. et al.(1996) "Tubulent cascades in foreign exchange markets" Nature 381767.

[26] Frisch, U. (1995) Turbulence: The Legacy of A.N. Kolmogorov, Cambridge University Press.

[27] Feller, W. (1950) Introduction to Probability theory and its applications, II, 3rd ed., John Wiley \& Sons, New York. 